\title{
Erratum to: The cognitive effects of chemotherapy in post- menopausal breast cancer patients: a controlled longitudinal study
}

\author{
Felice A. Tager • Paula S. McKinley • Freya R. Schnabel • Mahmoud El-Tamer • \\ Ying Keun K. Cheung • Yixin Fang • Claire R. Golden • Margery E. Frosch • \\ Ulya Habif • Margaret M. Mulligan · Ivy S. Chen · Dawn L. Hershman
}

Published online: 6 January 2010

(C) Springer Science+Business Media, LLC. 2010

\section{Erratum to: Breast Cancer Res Treat DOI 10.1007/s10549-009-0606-8}

In the paragraph starting with "Some investigators have..." Author would like to change "Heaton's Reliable Change Index" to "Reliable Change Index".

Also the corrected version of Table 4 is given below:
Table 4 Results of mixed model analyses in the five cognitive domains assessed

\begin{tabular}{llcl}
\hline & DF & $F$ value & $P$ value \\
\hline Motor & & & \\
Time & 2,422 & 0.20 & 0.82 \\
Chemotherapy & $1,57.9$ & 1.32 & 0.25 \\
Test & $3,54.7$ & 45.07 & $<0.001^{*}$ \\
Age & $1,58.9$ & 2.08 & 0.15 \\
Diagnosis & $2,58.3$ & 0.30 & 0.74 \\
Past HRT & $1,58.4$ & 0.43 & 0.51 \\
Time $\times$ chemotherapy & 2,422 & 5.01 & $0.01^{*}$ \\
\hline
\end{tabular}

The online version of the original article can be found under doi: 10.1007/s10549-009-0606-8.

F. A. Tager $(\bowtie) \cdot$ P. S. McKinley · C. R. Golden ·

M. E. Frosch · U. Habif · M. M. Mulligan · I. S. Chen

Behavioral Medicine Program, Department of Psychiatry,

College of Physicians and Surgeons, Columbia University

Medical Center, 1150 St. Nicholas Ave, Suite 1-121,

New York, NY, USA

e-mail: ft49@columbia.edu

\section{L. Hershman}

Department of Medicine, College of Physicians and Surgeons,

Columbia University Medical Center, New York, NY, USA

\section{El-Tamer}

Department of Surgical Oncology, College of Physicians and Surgeons, Columbia University Medical Center,

New York, NY, USA

F. A. Tager · P. S. McKinley · D. L. Hershman Herbert Irving Comprehensive Cancer Center, College of Physicians and Surgeons, Columbia University

Medical Center, New York, NY, USA
Y. K. K. Cheung · D. L. Hershman

Departments of Epidemiology and Biostatistics, Mailman School of Public Health, Columbia University Medical Center, New York, NY, USA

F. R. Schnabel

Department of Surgical Oncology, New York University Medical Center, New York, NY, USA

Y. Fang

Department of Mathematics and Statistics, Georgia State University, Atlanta, GA, USA 
Table 4 continued

\begin{tabular}{|c|c|c|c|}
\hline & DF & $F$ value & $P$ value \\
\hline \multicolumn{4}{|l|}{ Language } \\
\hline Time & 2,213 & 15.34 & $<0.001 *$ \\
\hline Chemotherapy & $1,60.1$ & 0.68 & 0.41 \\
\hline Test & $1,57.9$ & 11.21 & $0.001 *$ \\
\hline Age & $1,60.5$ & 1.25 & 0.27 \\
\hline Diagnosis & $2,60.3$ & 0.99 & 0.38 \\
\hline Past HRT & $1,60.2$ & 1.24 & 0.27 \\
\hline Time $\times$ test & 2,213 & 2.26 & 0.11 \\
\hline Time $\times$ chemotherapy & 2,213 & 0.87 & 0.42 \\
\hline \multicolumn{4}{|l|}{ Visuospatial } \\
\hline Time & 2,104 & 3.80 & $0.0256^{*}$ \\
\hline Chemotherapy & $1,49.3$ & 0.02 & 0.8821 \\
\hline Age & $1,50.7$ & 9.44 & $0.0034 *$ \\
\hline Diagnosis & $2,49.6$ & 1.45 & 0.2447 \\
\hline Past HRT & $1,49.6$ & 1.99 & 0.1644 \\
\hline Time $\times$ chemotherapy & 2,104 & 2.15 & 0.1211 \\
\hline \multicolumn{4}{|l|}{ Attention } \\
\hline Time & 2,673 & 0.45 & 0.6378 \\
\hline Chemotherapy & $1,60.4$ & 0.11 & 0.7413 \\
\hline Test & 5,56 & 3.43 & $0.0089^{*}$ \\
\hline Age & $1,60.5$ & 1.49 & 0.2263 \\
\hline Diagnosis & $2,60.5$ & 0.02 & 0.9799 \\
\hline Past HRT & $1,60.5$ & 0.45 & 0.5054 \\
\hline Time $\times$ chemotherapy & 2,673 & 0.67 & 0.5124 \\
\hline \multicolumn{4}{|l|}{ Memory } \\
\hline Time & 2,224 & 1.69 & 0.1863 \\
\hline Chemotherapy & $1,58.5$ & 2.97 & 0.0900 \\
\hline Test & $1,61.5$ & 9.31 & $0.0034 *$ \\
\hline Age & 1,59 & 0.04 & 0.8409 \\
\hline Diagnosis & $2,58.8$ & 0.29 & 0.7529 \\
\hline Past HRT & $1,58.8$ & 0.70 & 0.4066 \\
\hline Time $\times$ test & 2,225 & 3.81 & $0.0235^{*}$ \\
\hline Time $\times$ chemotherapy & 2,224 & 0.21 & 0.8071 \\
\hline
\end{tabular}

$* P<0.05$ 